\title{
The Research On The Relationship of APN with Cerebral Infarction
}

\author{
Zhen-wei $\mathrm{FAN}^{1, \mathrm{a}^{*}}$, Chunli Mei ${ }^{2, \mathrm{~b}}$ \\ ${ }^{1}$ College of Nursing, Beihua University,Jilin132013,China \\ ${ }^{2}$ College of Nursing, Beihua University,Jilin132013,China \\ a2289671@qq.com, b1227002496@qq.com
}

Keywords: APN Cerebral Infarction.

Abstract. Objective To investigate the relationships between the level of APN and cerebral infarction. Methods Measured APN、HDL-C、LDL-C、hs-CRP levels of 123 patients with cerebral infarction and 120 healthy controls, and analyzed the correlation with APN and the scale of clinical neurologic deficit. Results APN level in patient group was significantly lower than that of controls $(\mathrm{P}<0.01)$. With the Logistic regression analysis, there was a negative correction between the level of APN and the scale of clinical neurologic deficit in the patients, the lower level of APN and HDL-C, the higher level of LDL-C and hs-CRP are the risk factor of cerebral infarction. Conclusions The lower level of APN is the independent risk factor of cerebral infarction.

\section{Materials and Method}

\section{Study}

Infarction is a common and frequently-occurring disease of the nervous system, it is one of the major diseases which currently cause human death. Therefore, the study, on cerebral infarction risk factors, become more important. At present hypertension, as a risk factor for cerebral infarction, heart disease, diabetes, smoking, and hyperlipidemia have been confirmed. In recent years, some scholars have proposed that lowering serum adiponectin level was related to cerebral infarction. Now it is known that adiponectin,secreted by fat cells, between cells can interact with the extracellular matrix, is a specific protein and cytokines[1]. Some studies suggest that: adiponectin have some effects,such as reduce blood sugar, insulin sensitizers, inhibit atherosclerosis, anti-inflammatory etc. The aims of this paper is to study the relationship between serum adiponectin (APN) levels and cerebral infarction.

\section{Cerebral infarction group(CI group)}

Between January 2010 and December 2011,123 cases of patients,suffered infarction, were treated in neurology treatment area of Affiliated Hospital of BeiHua University.In this group,there are 63 cases of male patients, female patients 60 cases, with an average age of 63.2 years, all of the patients in accordance with the revised diagnostic criteria of Fourth National Conference on Cerebrovascular Disease,confirmed by CT or MRI of head, based on clinical neurological impairment score standards of fourth national cerebrovascular disease academic session, divided into three guoups:39 cases of light (0-15) grouping, 43 casesof medium (16-30) grouping,38 cases of heavy (31-45) grouping.

\section{The healthy control group}

120 cases of healthy control group were healthy,without a history of stroke and heart disease. In this group, there were 68 cases of male patients and 52 cases of female patients, with an average age of 62.8 years.

Two gruops of patients had no serious heart, liver, kidney, lung, blood and various infectious diseases. 


\section{Test Methods}

All patients were housed in fasting before 10:00 in blood day before yesterday evening, at 6:00 the next morning fasting,they were fasted venous blood $5 \mathrm{ml}$, until blood clotting, centrifugal separation of serum 10min,at 3500 / min speed. The part of the collected serum is placed in automatic biochemical analyzer, using reagents to measured high-density lipoprotein cholesterol(HDL-C), low-density lipoprotein cholesterol (LDL-C), high-sensitivity C-reactive protein (hs-CRP), strictly in accordance with the procedure is completed. Another part is placed in a refrigerator $-20{ }^{\circ} \mathrm{C}$ in storage, waiting for all of the samples have been collected,they were disposable to measured serum adiponectin. Serum adiponectin quantitative enzyme-linked immunosorbent assay (ELISA) method, The kit was purchased from Shenzhen Enoxaparin Gold Biotechnology Co., Ltd.

\section{statistical analysis}

Each set of data analysis with SPSS11.5 software package, all data are expressed as mean \pm standard deviation $\left(x_{ \pm s}\right)$, indicated and t-test, Logistic correlation analysis, test level $\alpha=0.05$.

\section{Results}

Compared with age and gender,CI group was not statistically significant differences(p>0.05) with the control group.To cerebral infarction patients, APN and HDL-C levels were significantly lower than the control group $(\mathrm{P}<0.01)$, LDL-C, hs-CRP was significantly higher than control group $(\mathrm{P}>0.01)$, As shown in Table 1. In neurological deficit scores as the dependent variable, In infarction group light, medium and heavy three types of adiponectin is independent variable to do correlation analysis, serum adiponectin and draw neurological deficit scores were negatively correlated $(\mathrm{p}<0.01)$, along with neurological deficit scores increased, serum adiponectin levels decreased (Table 2).

Tab1. Compared APN, HDL-C, LDL-C、 hs-CRP of cerebral infarction with controls

\begin{tabular}{cccccc}
\hline Group & $\mathrm{n}$ & $\begin{array}{c}\rho(\mathrm{APN}) / \\
(\mathrm{mg} . \mathrm{L}-1)\end{array}$ & $\begin{array}{c}\mathrm{c}(\text { HDL-C }) \\
/(\mathrm{mmol} . \mathrm{L}-1)\end{array}$ & $\begin{array}{c}\mathrm{c}(\mathrm{LDL}-\mathrm{C}) / \\
(\mathrm{mmol} . \mathrm{L}-1)\end{array}$ & $\begin{array}{c}\rho \text { (hs-CRP) } \\
/ \mathrm{mg} . \mathrm{m}-1)\end{array}$ \\
\hline $\begin{array}{c}\text { CI group } \\
\text { control group }\end{array}$ & 123 & $2.13 \pm 1.48$ & $0.58 \pm 0.21$ & $6.38 \pm 0.53$ & $12.13 \pm 1.65$ \\
\hline
\end{tabular}

Tab2 Compared with APN、 the scale of clinical neurologic deficit of every cerebral infarction group

\begin{tabular}{cccc}
\multicolumn{4}{c}{$\left(\bar{x}_{ \pm \mathrm{s}}\right)$} \\
\hline Group & $\mathrm{n}$ & $\rho($ APN $) /($ mg.L-1) & neurological deficit scores \\
\hline light & 39 & $3.23 \pm 0.38$ & $9.75 \pm 2.38$ \\
medium & 43 & $2.07 \pm 0.29$ & $23.74 \pm 3.85$ \\
heavy & 38 & $0.96 \pm 0.31$ & $36.27 \pm 4.24$ \\
\hline
\end{tabular}

\section{Discussion}

Adiponectin is the only negative regulation hormones of all adipocytokine.It's clearly the current number of amino acids, make up of adiponectin, is 244 .Its molecular weight is $30 \times 103$, the gene encoding existed in the human body, which can controls ADP, located in 3q27 locus. The present study showed that adiponectin levels are closely related with numerous heart, cerebrovascular disease risk factors, such as gender, obesity, high blood sugar, high cholesterol, high blood pressure.At present,it is not clear in the body of specific functions and mechanisms of action, but by the experimental animal model of atherosclerosis conclude:serum adiponectin levels Lower,triglycerides and low-density lipoprotein elevated, high density lipoprotein decreases and vice versa; adiponectin 
concentration inhibits triglyceride levels elevated and enhances insulin action to inhibit glycogen gluconeogenesis. In this study,compared adiponectin, HDL and LDL levels of the patients,sufferred cerebral infarction, with the measurement of the control group, conclusions consistent with the above result.

Atherosclerosis is an important cause of cerebral infarction, adiponectin have an affect on the occurrence of atherosclerosis and development,by inhibiting inflammation, inhibiting the proliferation and migration of vascular smooth muscle, improving lipid metabolism disorders and other aspects.

\section{inhibit the inflammatory response}

When adiponectin deposited on the damaged arterial wall,it may inhibit endothelial inflammatory response[4]. Ouchi et al [5] found that adiponectin can reduce the adhesion of monocytes, incidence of early atherosclerosis in the blood vessel wall damage, even THP-1 cells in human aortic endothelial tissue arranged in rows, while adiponectin,by inhibiting lipid accumulation and expression in class A scavenger receptor on macrophages,reduce the conversion of macrophages into foam cells. Currently, it is recognized TNF- $\alpha$ is cytokines, secreted by macrophages, T lymphocytes, fibroblasts, fat cells, not only have multiple biological functions, but also regulate the body's inflammatory response and immune response [6]. Some study found: TNF-- $\alpha$ can enhance monocyte adhesion of endothelial cells, promote transformation from macrophage to foam cell, while the normal concentration of APN inhibit TNF- $\alpha$ in the above-mentioned effects, it follows: in the normal human body, APN inhibits monocyte adhesion to endothelial cells andtransformation from macrophages to foam cells [7]. In vitro, APN inhibition of TNF- $\alpha$ is being, and dose-dependent manner[5]. At the same time, fat cells express APN gene was inhibited by TNF- $\alpha[8]$, we can see the existence of antagonistic relations, eventually,the vicious cycle of fat cells and macrophages established, the two Expression levels of TNF- $\alpha$ and adiponectin of obese people was negatively correlated.

\section{inhibit the proliferation and migration of vascular smooth muscle}

Cell Culture [9] demonstrated: APN inhibit differentiation of hematopoietic cells, inhibit proliferation and differentiation of arterial endothelial cell and migration of skin damage zone inwardly.Arterial intimal thickening and medial smooth muscle cell proliferation and migration to the under of intima, are considered to be another mechanism of occurrence and development of atherosclerosis. From this: in atherosclerosis development and progression, the platelet-derived growth factor (PDGF) or heparin-binding epidermal growth factor (HB-EGF) induced angiogenesis smooth muscle cell proliferation plays an important role, adiponectin on atherosclerosis have a certain extent.

\section{improve lipid metabolism disorders}

Adiponectin improves hepatic insulin sensitivity, so that an increase in fatty acid oxidation in peripheral tissues, VLDL synthesis decreased, thus ultimately improving lipid metabolism [10]. The study for transgenic mice found that: clearing plasma FFA delayed is the result of adiponectin missing[11]. It was suggested that there is an adjustment of atherosclerosis occurrence and development of fat - vascular regulation, and adiponectin axis may be the bridge between them[12]. In addition, adiponectin also has an effect on outer improved extracellular matrix degradation and stable atherosclerotic plaque.

\section{Summary}

In conclusion,the adiponectin has effect on anti-inflammatory and anti-atherosclerotic, this is consistent with the results of Jilong Gtuo et al [13]. From this experiment further confirmed in the clinic, adiponectin may affect the occurrence and development of cerebral infarction, cerebral 
infarction can be used as an independent risk factor. It is clinically provides another possible way of cerebral intervention.

\section{References}

[1]Yoda - Murakam iM, Tan iguch iM, Takahash iK, et al Change in expression of GBP28 / adi ponectin in carbon tetrachloride administrated mouse liver[J].Biochem Biophys Res Commun,2001;285(2):372 -377.

[2]Weihong Wu,Jingchen Zheng. Adiponectin and lipid metabolism[J]. General Practice 2007;5(6): 541-542.

[3]Fourth National Conference on cerebrovascular disease standards(1995)[J].Journal of Neurology,1996;29(6):376-383.

[4]Matsuda M,Shimomural,SataM,et al.Role of adponectin in preventing vascular stenosis. The missing link of adipo-vascularaxis[J].J Biol Chem,2002;277(40): 37487-37491.

[5]Ouchi N, Kihara S, Arita Y, et al. Adipocyte-derived plasma protein adiponectin, suppresses lipid accumulation and class a scavenger receptor expression in human monocyte-derived macrophages[J]. Circulation, 2001;103:1057-1063.

[6]Hotarnisigil G S, Shargill N S, Spiegel man B M. Adipose expression of tumor necrosis factor-alpha: direct role in obesity-linked insulin resistance[J]. Science, 1993;259:87.

[7]Qiang Pei, Yongwen Qin, etc. Relationship between adiponectin levels and coronary lesion severity of serum lipids[J].Journal of Clinical Cardiology2004;20 (10):619-621.

[8]Fasshauer M, Klein J, Neumann S, et al. Hormonal regulation of adiponectin gene express in 3T3-L1 adipocytes[J]. Biochem Biophys Res Commun, 2002;290:1084 1089.

[9]Shibata R,Sate K,Kumada M,et a1 . Adiponectin accumulates in myocardial tissue that has been damaged by ischemia-reperfusion injury via leakage from the VasClllar compartment.Cardiovasc Res,2007;74(3):471-479.

[10]Chao Liu. Retrospect and Prospect of metabolic syndrome[J]. Chinese Journal of Internal Medicine 2006; 26 (20): :1581-1584.

[11]Zhengzhao Deng, Rongli Qian.Abnormal lipid metabolism and diabetes treatment[J]. Chinese Journal of Diabetes2001; 9 (4): 251-252.

[12]Yan Liu, Dajin Zou.Protective effects of adiponectin in atherosclerosis[J].Chinese Journal of Clinical Rehabilitation2003; 7 (21):2969- 2970.

[13]Jilong Guo,Qing Yuan,Yangguo Ma.Relationship between serum adiponectin levels and cerebral infarction[J].Laboratory Medicine2009; 24(2):140 -142. 